\title{
Subgrouping of Japanese middle-aged women attending a menopause clinic using physical and psychological symptom profiles: a cross-sectional study
}

Masakazu Terauchi ${ }^{1 *}$, Asuka Hirose $^{2}$, Mihoko Akiyoshi², Yoko Owa², Kiyoko Kato $^{2}$ and Toshiro Kubota²

\begin{abstract}
Background: Women in the menopausal transition and the postmenopausal period are affected with vasomotor symptoms, urogenital atrophy, sexual dysfunction, somatic symptoms, cognitive difficulty, sleep disturbance, and psychological problems. It is important to gain a better understanding of the complexity and diversity of climacteric disturbance in order to optimize treatments for individual patients. The aim of this study was to identify subgroups of Japanese perimenopausal and postmenopausal women attending a menopause clinic based on their physical and psychological symptom profiles.
\end{abstract}

Methods: We administered the Menopausal Health-Related Quality of Life questionnaire to 491 Japanese women aged 40-64 years who had enrolled in the Systematic Health and Nutrition Education Program at the Menopause Clinic of the Tokyo Medical and Dental University Hospital between 2005 and 2012. We performed a principal component analysis followed by a hierarchical cluster analysis of the responses to 9 physical and 12 psychological items on the questionnaire.

Results: The first analysis extracted 3 principal components that defined the variance of physical and psychological symptom profiles: depression, somatic, and vasomotor/sleep. A subsequent cluster analysis was performed based on the 3 principal components to generate 4 clusters, CL8 ( $N=162 ; 33.0 \%), C L 6(N=111 ; 22.6 \%), C L 5$ ( $N=102$; 20.8\%), and CL4 ( $N=116 ; 23.6 \%)$. CL8 included women who only had mild-to-moderate musculoskeletal pains and tiredness. All women in CL6, CL5, and CL4 described their musculoskeletal pains and tiredness as moderate to severe. The women in CL5 also had moderate-to-severe vasomotor symptoms, while the women in CL4 also suffered from moderate-to-severe psychological symptoms, such as depression, anxiety, and insomnia.

Conclusions: Distinct subgroups of Japanese perimenopausal and postmenopausal women were identified based on their symptom profiles. Menopausal symptoms were shown to accumulate in this population in the order of musculoskeletal pains and tiredness, vasomotor symptoms, and psychological symptoms.

Keywords: Principal component analysis, Cluster analysis, Musculoskeletal pains, Tiredness, Vasomotor symptoms, Depression, Anxiety, Insomnia

\footnotetext{
* Correspondence: teragyne@tmd.ac.jp

'Department of Women's Health, Tokyo Medical and Dental University,

Yushima 1-5-45, Bunkyo, Tokyo 113-8510, Japan

Full list of author information is available at the end of the article
} 


\section{Background}

Women in the menopausal transition and the postmenopausal period are affected with vasomotor symptoms, urogenital atrophy, sexual dysfunction, somatic symptoms, cognitive difficulty, sleep disturbance, and psychological problems. Some of these effects, particularly vasomotor symptoms and urogenital atrophy, are closely associated with estrogen deficiency, whereas the exact mechanism underlying the other symptoms is not fully understood.

Therefore, it is important to gain a better understanding of the complexity and diversity of climacteric disturbance in order to optimize treatments for individual patients. Accordingly, previous trials have categorized menopausal symptoms of non-menopause clinic samples [1] and population-based samples [2-8], and identified subgroups of menopausal women on the basis of their responses to symptom checklists. Yet, subgrouping of menopause clinic samples, which would be most relevant to clinical practice, has not been reported.

The aim of the present study was to identify subgroups of Japanese perimenopausal and postmenopausal women attending a menopause clinic based on their physical and psychological symptom profiles.

\section{Methods}

In this cross-sectional study, we examined the medical records of a study population similar to that analyzed in our previous studies [9-15]. Specifically, we retrospectively analyzed the first-visit records of 491 Japanese perimenopausal and postmenopausal women aged 40-64 years who had enrolled in the Systematic Health and Nutrition Education Program conducted at the Menopause Clinic of the Tokyo Medical and Dental University Hospital between 2005 and 2012. All middle-aged women who had enrolled in this program were referred to our clinic for the treatment of their menopausal symptoms, and all participants provided informed consent. Before beginning our investigation, we obtained approval for the study protocol from the Tokyo Medical and Dental University Review Board and confirmed that all participants provided informed consent. We ensured that all study procedures were implemented in accordance with the Declaration of Helsinki.

A woman was defined as being in the menopausal transition if she menstruated within the past 12 months but had either missed a period or had experienced irregular cycles in the past 3 months; a woman was defined as being postmenopausal if she had not menstruated in the past 12 months [16]. At their initial visit, women were interviewed by physicians and nutritionists, and they provided data regarding their menopausal symptoms and quality of life during the past month by completing the Menopausal Health-Related Quality of Life questionnaire, which we developed and validated at our clinic [9-15,17]. The Menopausal Health-Related Quality of Life questionnaire is a modification of the Women's Health Questionnaire developed by Hunter et al. and contains 38 items scored on a 4-point or binary scale covering 4 major domains (physical health, mental health, life satisfaction, and social involvement) of a woman's health during the menopausal transition $[1,18]$.

The items used to assess the 2 domains of interest in the current study, physical health and mental health, are shown in Table 1 . The physical health domain comprised 9 items that assessed somatic and vasomotor symptoms, and the mental health domain comprised 12 items that assessed depressed mood, cognitive difficulties, anxiety and fears, sexual functioning, and sleep problems. For convenience, the scoring system shown in Table 1 is in

Table 1 Physical and mental health domain items in the Menopausal Health-Related Quality of Life questionnaire

\begin{tabular}{lllll}
\hline $0-1$ times & $1-2$ times & 3-4 times & Almost \\
a month & a week & per week & every day \\
(none) & (mild) & (moderate) & (severe)
\end{tabular}

\begin{tabular}{|c|c|c|c|c|}
\hline \multirow{2}{*}{\multicolumn{5}{|c|}{ Physical health domain }} \\
\hline & & & & \\
\hline Nausea & 0 & 1 & 2 & 3 \\
\hline Dizziness & 0 & 1 & 2 & 3 \\
\hline Numbness & 0 & 1 & 2 & 3 \\
\hline $\begin{array}{l}\text { Muscle and joint } \\
\text { pains }\end{array}$ & 0 & 1 & 2 & 3 \\
\hline Tiredness & 0 & 1 & 2 & 3 \\
\hline Headaches & 0 & 1 & 2 & 3 \\
\hline Frequent urination & 0 & 1 & 2 & 3 \\
\hline Hot flush & 0 & 1 & 2 & 3 \\
\hline Night sweats & 0 & 1 & 2 & 3 \\
\hline \multicolumn{5}{|l|}{ Mental health domain } \\
\hline $\begin{array}{l}\text { Loss of interest in } \\
\text { things }\end{array}$ & 0 & 1 & 2 & 3 \\
\hline Lack of enjoyment & 0 & 1 & 2 & 3 \\
\hline Low energy & 0 & 1 & 2 & 3 \\
\hline Depressed mood & 0 & 1 & 2 & 3 \\
\hline Poor memory & 0 & 1 & 2 & 3 \\
\hline $\begin{array}{l}\text { Difficulty in } \\
\text { concentration }\end{array}$ & 0 & 1 & 2 & 3 \\
\hline $\begin{array}{l}\text { Frightened/panicky } \\
\text { feelings }\end{array}$ & 0 & 1 & 2 & 3 \\
\hline Feel tense/wound up & 0 & 1 & 2 & 3 \\
\hline $\begin{array}{l}\text { Dissatisfaction with } \\
\text { sexual relationship }\end{array}$ & 0 & 1 & 2 & 3 \\
\hline $\begin{array}{l}\text { Difficulty in } \\
\text { initiating sleep }\end{array}$ & 0 & 1 & 2 & 3 \\
\hline $\begin{array}{l}\text { Non-restorative } \\
\text { sleep }\end{array}$ & 0 & 1 & 2 & 3 \\
\hline Low self-esteem & 0 & 1 & 2 & 3 \\
\hline
\end{tabular}


the reverse order to that used in the quality of life questionnaire in our clinical practice; higher scores indicate worse physical and mental functioning.

The symptom score profiles of participants were subjected to a principal component analysis (PCA) to examine the relationships among the 21 physical and mental symptoms and to define factors (principal components [PCs]), composed of scores of related symptoms, that could account for most of the variance in profiles of symptom scores. The appropriate number of PCs was determined from the point on the Scree plot where eigenvalues leveled off. PC values for each woman were then calculated based on the formulas obtained from the PCA.

Subsequently, a hierarchical cluster analysis was performed using Ward's minimum variance technique to subgroup women based on the Euclidean distances among the PC values.

All statistical analyses were performed with SAS 9.2 (SAS Institute, Cary, NC, USA). $P<0.05$ was defined as statistically significant.

\section{Results}

A total of 491 women were included in the study, and the mean \pm standard deviation age of participants was $51.8 \pm 5.4$ years; $44 \%$ of participants were classified as being in the menopausal transition, and 56\% were classified as postmenopausal. The eigenvalues, percent of variance, and factor loading (eigenvectors) obtained from the initial PCA of study participants' symptom score profiles are shown in Table 2. Three PCs were identified, accounting for $48 \%$ of variance. The first PC (PC1) accounted for $32.8 \%$ of variance, for which the 3 symptom scores with largest eigenvectors were low energy,

Table 2 Principal component analysis

\begin{tabular}{llll}
\hline & Eigenvalue & \% of variance & Eigenvector \\
\hline Principal component 1: & 6.89 & 32.8 & \\
"depression" & & \\
Low energy & & 0.321 \\
Depressed mood & & 0.318 \\
Lack of enjoyment & & 0.302 \\
Principal component 2: & 1.91 & 9.1 & \\
"somatic" & & \\
Dizziness & & 0.371 \\
Muscle and joint pains & & 0.320 \\
Nausea & & 0.318 \\
Principal component 3: & 1.28 & 6.1 & \\
"vasomotor/sleep" & & \\
Night sweats & & 0.434 \\
Difficulty in initiating sleep & & 0.318 \\
Hot flush & & \\
\hline
\end{tabular}

depressed mood, and lack of enjoyment. Therefore, PC1 was best characterized as the component for "depression". Likewise, PC2, accounting for 9.1\% of variance, was characterized as "somatic" because its top 3 symptoms were dizziness, muscle and joint pain, and nausea. PC3, which accounted for $6.1 \%$ of variance, was characterized as "vasomotor/sleep", as symptoms included night sweats, difficulty in initiating sleep, and hot flush. Based on the PCA, PC1, 2, and 3 for each woman was calculated as follows:

$$
\begin{aligned}
\text { PC1 }= & 0.321 * \text { "low energy" score }+ \\
& 0.318 * \text { "depressed mood" score }+ \\
& 0.302 * \text { "lack of enjoyment" score } \\
\text { PC2 }= & 0.371 * \text { "dizziness" score }+ \\
& 0.320 * \text { "muscle and joint pains" score }+ \\
& 0.318 * \text { "nausea" score } \\
\text { PC3 }= & 0.434 * \text { "night sweats" score }+ \\
& 0.318 * \text { "difficulty in initiating sleep" score }+ \\
& 0.317 * \text { "hot flush" score }
\end{aligned}
$$

Four distinct clusters (CLs) were obtained from the subsequent cluster analysis based on the Euclidean distances among the PC values: CL8 ( $\mathrm{N}=162 ; 33.0 \%)$, CL6 $(\mathrm{N}=111$; $22.6 \%)$, CL5 ( $\mathrm{N}=102 ; 20.8 \%)$, and CL4 $(\mathrm{N}=116 ; 23.6 \%)$ (Figure 1).

Baseline physical characteristics of the study participants by cluster are shown in Table 3. One-way analysis of variance and a chi-square test showed that height, waist-hip ratio, systolic/diastolic pressure, pulse rate, and the proportions of women who smoked and exercised regularly differed among the groups. The average physical and psychological symptom scores of the 4 clusters are shown in Table 4. The Kruskal-Wallis test showed that scores for every item were significantly different among the groups. On the basis of the average score for each item, the 4 clusters were characterized as follows: CL8 was composed of women who only had mild-tomoderate muscle and joint pain and tiredness. All the women in CL6, CL5, and CL4 assessed their musculoskeletal pains and tiredness as moderate to severe. The women in CL5 also had moderate-to-severe vasomotor symptoms, while the women in CL4 suffered from moderate-to-severe psychological symptoms, such as depression, anxiety, and insomnia.

Women with more severe symptoms tended to have higher systolic/diastolic pressure, higher pulse rates, smoked more, and exercised less frequently than women with milder symptoms (Table 3 ). 


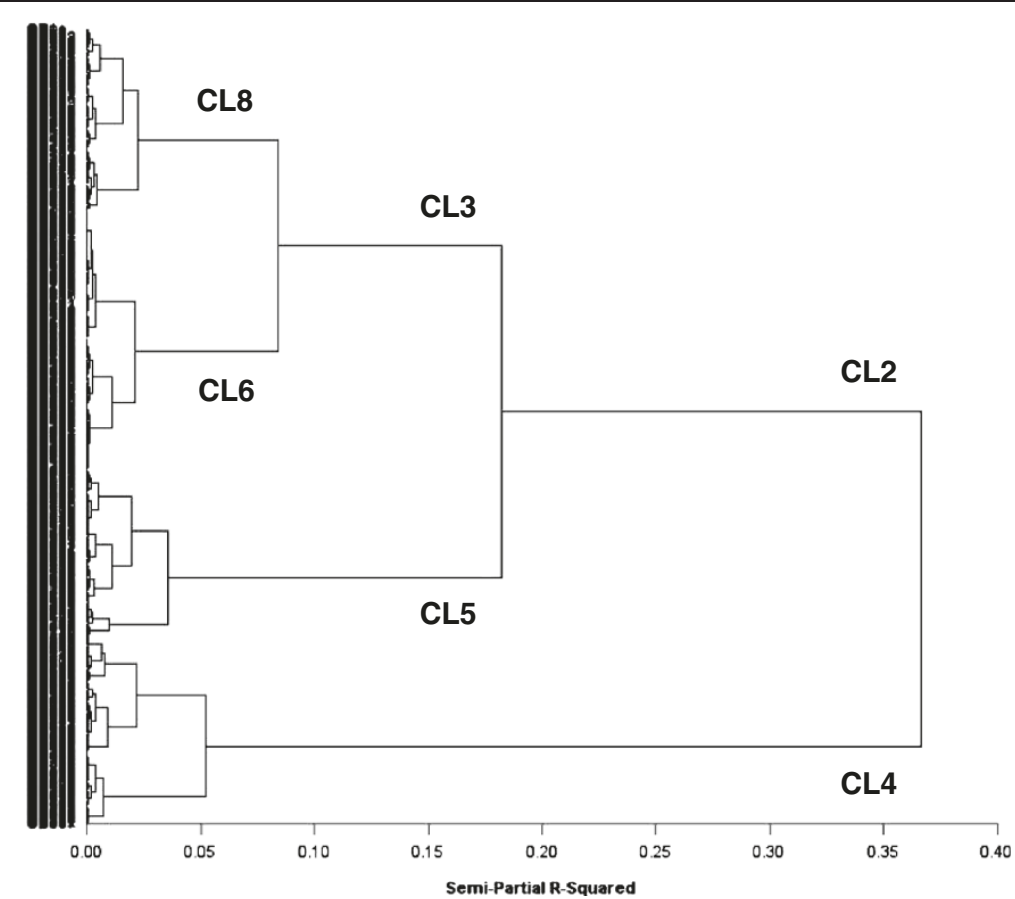

Figure 1 Cluster analysis. Four distinct clusters (CLs) were obtained from the subsequent cluster analysis based on the Euclidean distances among the PC values: CL8 (N=162; 33.0\%), CL6 (N=111; 22.6\%), CL5 (N=102; 20.8\%), and CL4 ( $N=116 ; 23.6 \%)$.

Table 3 Baseline physical characteristics of the study participants $(\mathrm{N}=491)$

\begin{tabular}{|c|c|c|c|c|c|}
\hline & $\begin{array}{l}\text { CL8 } \\
(\mathrm{N}=162)\end{array}$ & $\begin{array}{l}\text { CL6 } \\
(\mathrm{N}=111)\end{array}$ & $\begin{array}{l}\text { CL5 } \\
(\mathrm{N}=102)\end{array}$ & $\begin{array}{l}\text { CL4 } \\
(\mathrm{N}=116)\end{array}$ & $P$ \\
\hline Age (years) & $52.1 \pm 6.0$ & $51.0 \pm 5.5$ & $51.5 \pm 5.1$ & $52.5 \pm 4.8$ & 0.205 \\
\hline Perimenopausal/postmenopausal (\%) & $40.3 / 59.7$ & $49.1 / 50.9$ & $46.5 / 53.5$ & $41.4 / 58.6$ & 0.455 \\
\hline Height $(\mathrm{cm})$ & $156.4 \pm 5.6$ & $158.1 \pm 5.3$ & $157.1 \pm 5.4$ & $155.5 \pm 5.4$ & 0.004 \\
\hline Body weight (kg) & $53.0 \pm 8.5$ & $53.0 \pm 8.7$ & $54.1 \pm 9.9$ & $51.9 \pm 9.4$ & 0.337 \\
\hline Body mass index $\left(\mathrm{kg} \cdot \mathrm{m}^{-2}\right)$ & $21.6 \pm 3.3$ & $21.2 \pm 3.3$ & $21.9 \pm 3.7$ & $21.4 \pm 3.6$ & 0.491 \\
\hline Body fat (\%) & $26.7 \pm 7.3$ & $26.9 \pm 8.4$ & $27.6 \pm 7.8$ & $26.4 \pm 7.8$ & 0.806 \\
\hline Muscle mass (kg) & $35.9 \pm 2.7$ & $36.0 \pm 2.8$ & $36.2 \pm 3.4$ & $35.3 \pm 3.0$ & 0.220 \\
\hline Water mass (kg) & $27.5 \pm 2.6$ & $27.3 \pm 2.9$ & $27.8 \pm 3.5$ & $26.9 \pm 2.8$ & 0.211 \\
\hline Waist circumference $(\mathrm{cm})$ & $78.8 \pm 9.0$ & $77.2 \pm 9.5$ & $80.1 \pm 9.8$ & $77.6 \pm 10.2$ & 0.516 \\
\hline Hip circumference (cm) & $92.0 \pm 5.8$ & $91.1 \pm 6.2$ & $91.5 \pm 6.1$ & $90.5 \pm 7.2$ & 0.295 \\
\hline Waist-hip ratio (\%) & $85.5 \pm 6.0$ & $84.5 \pm 6.3$ & $87.4 \pm 6.4$ & $85.6 \pm 6.8$ & 0.013 \\
\hline Systolic pressure $(\mathrm{mmHg})$ & $122.2 \pm 14.5$ & $124.1 \pm 17.9$ & $126.8 \pm 14.9$ & $127.7 \pm 16.8$ & 0.021 \\
\hline Diastolic pressure $(\mathrm{mmHg})$ & $78.5 \pm 10.1$ & $78.6 \pm 12.2$ & $81.8 \pm 11.0$ & $82.3 \pm 11.6$ & 0.007 \\
\hline Pulse rate $\left(\mathrm{min}^{-1}\right)$ & $61.7 \pm 9.6$ & $62.2 \pm 8.0$ & $64.2 \pm 10.9$ & $65.4 \pm 12.6$ & 0.015 \\
\hline Cardio-ankle vascular index & $7.5 \pm 0.8$ & $7.5 \pm 0.8$ & $7.5 \pm 0.8$ & $7.5 \pm 0.7$ & 0.944 \\
\hline Daily alcohol consumption (\%) & 8.6 & 10.9 & 17.5 & 10.6 & 0.184 \\
\hline Smoking (\%) & 6.6 & 12.7 & 17.2 & 15.9 & 0.046 \\
\hline Regular exercise (\%) & 52.2 & 39.4 & 51.0 & 27.8 & $<0.001$ \\
\hline
\end{tabular}

Data are expressed as mean \pm standard deviation.

Statistical analyses were performed using one-way analysis of variance and chi-square tests. 
Table 4 Average physical and psychological symptom scores of the 4 clusters $(N=491)$

\begin{tabular}{|c|c|c|c|c|c|}
\hline & $\begin{array}{l}\text { CL8 } \\
(N=162)\end{array}$ & $\begin{array}{l}\text { CL6 } \\
(\mathrm{N}=111)\end{array}$ & $\begin{array}{l}\text { CL5 } \\
(N=102)\end{array}$ & $\begin{array}{l}\text { CL4 } \\
(\mathrm{N}=116)\end{array}$ & $P$ \\
\hline \multicolumn{6}{|l|}{ Physical health domain } \\
\hline Nausea & $0.0 \pm 0.2$ & $0.3 \pm 0.7$ & $0.5 \pm 1.0$ & $0.6 \pm 1.0$ & $<0.0001$ \\
\hline Dizziness & $0.2 \pm 0.5$ & $0.7 \pm 0.9$ & $0.6 \pm 1.0$ & $0.7 \pm 1.0$ & $<0.0001$ \\
\hline Numbness & $0.4 \pm 1.0$ & $0.8 \pm 1.1$ & $0.8 \pm 1.2$ & $1.1 \pm 1.3$ & $<0.0001$ \\
\hline Muscle and joint pains & $1.6 \pm 1.2$ & $2.3 \pm 1.0$ & $2.2 \pm 1.1$ & $2.3 \pm 1.1$ & $<0.0001$ \\
\hline Tiredness & $1.0 \pm 1.0$ & $2.2 \pm 0.9$ & $2.0 \pm 1.1$ & $2.6 \pm 0.9$ & $<0.0001$ \\
\hline Headaches & $0.4 \pm 0.7$ & $0.8 \pm 1.0$ & $1.0 \pm 1.1$ & $1.3 \pm 1.2$ & $<0.0001$ \\
\hline Frequent Urination & $0.6 \pm 1.0$ & $0.9 \pm 1.2$ & $1.3 \pm 1.3$ & $1.3 \pm 1.4$ & $<0.0001$ \\
\hline Hot flush & $0.5 \pm 0.9$ & $0.6 \pm 0.9$ & $2.1 \pm 1.1$ & $1.4 \pm 1.2$ & $<0.0001$ \\
\hline Night sweats & $0.1 \pm 0.4$ & $0.2 \pm 0.5$ & $2.0 \pm 1.1$ & $1.3 \pm 1.2$ & $<0.0001$ \\
\hline \multicolumn{6}{|l|}{ Mental health domain } \\
\hline Loss of interest in things & $0.1 \pm 0.4$ & $0.7 \pm 0.9$ & $0.7 \pm 0.8$ & $2.2 \pm 1.1$ & $<0.0001$ \\
\hline Lack of enjoyment & $0.0 \pm 0.2$ & $0.8 \pm 0.7$ & $0.5 \pm 0.6$ & $2.6 \pm 0.8$ & $<0.0001$ \\
\hline Low energy & $0.1 \pm 0.3$ & $1.3 \pm 0.7$ & $0.8 \pm 0.8$ & $2.8 \pm 0.5$ & $<0.0001$ \\
\hline Depressed mood & $0.2 \pm 0.4$ & $1.5 \pm 0.8$ & $0.9 \pm 0.8$ & $2.9 \pm 0.4$ & $<0.0001$ \\
\hline Poor memory & $0.8 \pm 0.9$ & $1.3 \pm 1.0$ & $1.3 \pm 1.0$ & $1.7 \pm 1.1$ & $<0.0001$ \\
\hline Difficulty in concentration & $0.8 \pm 0.9$ & $1.3 \pm 1.0$ & $1.3 \pm 1.0$ & $1.7 \pm 1.1$ & $<0.0001$ \\
\hline Frightened/panicky feelings & $0.3 \pm 0.6$ & $1.3 \pm 1.0$ & $1.1 \pm 0.9$ & $2.2 \pm 1.0$ & $<0.0001$ \\
\hline Feel tense/wound up & $0.3 \pm 0.6$ & $0.8 \pm 1.0$ & $0.9 \pm 1.1$ & $1.8 \pm 1.2$ & $<0.0001$ \\
\hline Dissatisfaction with sexual relationship & $0.1 \pm 0.5$ & $0.3 \pm 0.7$ & $0.4 \pm 0.9$ & $0.4 \pm 0.9$ & 0.0134 \\
\hline Difficulty in initiating sleep & $0.4 \pm 0.7$ & $0.8 \pm 1.0$ & $1.6 \pm 1.2$ & $1.7 \pm 1.3$ & $<0.0001$ \\
\hline Non-restorative sleep & $0.5 \pm 0.9$ & $1.3 \pm 1.2$ & $1.7 \pm 1.2$ & $2.0 \pm 1.1$ & $<0.0001$ \\
\hline Low self-esteem & $0.5 \pm 0.8$ & $1.4 \pm 1.2$ & $1.1 \pm 1.1$ & $2.1 \pm 1.2$ & $<0.0001$ \\
\hline
\end{tabular}

Data are expressed as mean \pm standard deviation.

Statistical analyses were performed using the Kruskal-Wallis test.

\section{Discussion}

In this study, we analyzed the responses to 9 physical and 12 psychological items on the Menopausal HealthRelated Quality of Life questionnaire by 491 Japanese women attending our menopause clinic aged 40-64 years. The first analysis extracted 3 PCs (depression, somatic, and vasomotor/sleep) that defined the variance of physical and psychological symptom profiles of the women. A subsequent cluster analysis was performed based on the 3 PCs to generate 4 clusters: CL8, CL6, CL5, and CL4. CL8 was composed of women who had only mild-to-moderate musculoskeletal pains and tiredness. All women in CL6, CL5, and CL4 described their musculoskeletal pains and tiredness as moderate to severe. The women in CL5 also had moderate-to-severe vasomotor symptoms, while the women in CL4 also suffered from moderate-to-severe psychological symptoms, such as depression, anxiety, and insomnia.

Many previous studies have categorized menopausal symptoms of non-menopause clinic samples and population based samples, and identified subgroups of women in the menopausal transition on the basis of their responses to symptom checklists. In 1986, Hunter et al. first introduced their "Women's Health Questionnaire", and they performed a PCA of responses to the 36-item symptom checklist among 682 English women attending an ovarian screening program aged 45-65 years [1]. Based on the analysis, they defined 9 symptom clusters, namely "somatic symptoms", "depressed mood", "cognitive difficulties", "anxiety/fears", "sexual functioning", "vasomotor symptoms", "sleep problems", "menstrual", and "attractive".

Similar studies using PCA methods have been performed to identify menopausal symptom clusters specific to Hong Kong Chinese [2] and Taiwanese [3]; to examine cross-cultural variation among the United States, Spain, Lebanon, and Morocco [4]; to investigate how symptoms cluster across menopausal transition stages [5]; and to determine which symptom cluster has the greatest effect on quality of life [6]. In a random telephone survey of Hong Kong Chinese women, Ho et al. identified 5 symptom clusters: psychological, musculoskeletal/gastrointestinal, non-specific somatic, respiratory, and vasomotor 
[2]; in a resident cohort study of Taiwanese women, Fuh et al. identified 4 symptom clusters: musculoskeletal, non-specific somatic complaints, urogenital, and vasomotor [3]. Sievert et al. showed in their study of general population that the inter-correlation among symptoms differed in country-specific ways; for example, hot flush grouped with vaginal dryness and sexual symptoms in Spain, with general somatic symptoms in Morocco, and did not cluster with other symptoms in the United States or Lebanon [4]. Based on the observations from the Seattle Midlife Women's Health Study (SMWHS), Cray et al. revealed similar factor structures across the 4 menopausal transition stages (late reproductive, early or late menopausal transition, or early postmenopause) in that each stage revealed a mood component, a vasomotor component, and a pain component [5]. A study by Greenblum et al. identified 3 symptom clusters in middle-aged Floridian community-dwelling women: (1) anxiety, irritability, and fatigue; (2) weight gain and urinary stress incontinence; and (3) vaginal dryness and sleep disturbances, which had the greatest impact on quality of life [6].

A different approach using cluster analysis was taken to investigate the relationships between patterns of depressed mood and menopausal transition stages [19] or to identify clusters of female urological symptoms [20]. Using the data from SMWHS, Woods et al. revealed that the majority of women experienced the menopausal transition without a high severity of depressed mood, while a small group of women had mood worsening over time and others improved [19]. Hall et al. conducted a cluster analysis of urological symptoms among women in the Boston Area Community Health Survey and identified 4 clusters, which were distinguished by the severity of storage symptoms, frequency symptoms, urinary incontinence, and interference [20].

Recently, Cray et al. introduced latent class analysis to identify subgroups of women in the menopausal transition stage that experienced the same cluster of representative symptoms $[7,8]$. They first selected 5 symptoms based on PCA of the SMWHS participants' responses to the 47-item symptom checklist: sleep, pain, mood, cognitive, and tension. Multilevel latent class analysis using scores for hot flush and these 5 symptoms identified 3 classes: low severity levels for all symptoms; low severity hot flush and moderate severity levels for all other symptom factors; high severity hot flush with lower severity levels of all other symptom factors. In their most recent report, Woods et al. identified several endocrine biomarkers associated with the distinction among the subgroups, including estrogen, follicle stimulating hormone, epinephrine, and norepinephrine [21].

Similar to our findings, a 2013 SMWHS report by Cray et al. identified 3 major components of symptoms identified by PCA [5]. These included psychological, vasomotor, and musculoskeletal pains, of which the psychological component accounted for more than $30 \%$ of variance of the profile of menopausal symptoms. Cross-cultural diversity in menopausal symptomatology has been frequently demonstrated [22], and fewer Asian women have been shown to report vasomotor and other menopausal symptoms compared with their Caucasoid counterparts [23]; nevertheless, these fundamental components might be similar between the United States and Japan.

One of the major limitations of our study was that the participants were recruited in a clinical setting, and the results were not necessarily representative of the general population of Japan. Nevertheless, the findings of the current study are relevant to clinical practice as they address the complex symptomatology in women attending a menopause clinic.

\section{Conclusions}

In conclusion, distinct subgroups of Japanese perimenopausal and postmenopausal women were identified based on the severity of depression, somatic, and vasomotor/ sleep symptoms. This subgrouping of women revealed that menopausal symptoms accumulate in this population in the order of musculoskeletal pains, vasomotor symptoms, and psychological symptoms.

\section{Competing interests \\ The authors declare that they have no competing interest.}

\section{Authors' contributions}

MT conceived of and designed the study, analyzed and interpreted the data, and drafted the manuscript. AH, MA, YO, KK, and TK participated in the acquisition of the data. All authors read and approved the final manuscript.

\section{Acknowledgement}

We thank all the women who participated in the Systematic Health and Nutrition Education Program at the Menopause Clinic of the Tokyo Medical and Dental University Hospital.

\section{Author details}

'Department of Women's Health, Tokyo Medical and Dental University, Yushima 1-5-45, Bunkyo, Tokyo 113-8510, Japan. ${ }^{2}$ Department of Obstetrics and Gynecology, Tokyo Medical and Dental University, Yushima 1-5-45, Bunkyo, Tokyo 113-8510, Japan.

Received: 23 August 2014 Accepted: 10 November 2014

Published online: 25 November 2014

\section{References}

1. Hunter M, Battersby R, Whitehead M: Relationships between psychological symptoms, somatic complaints and menopausal status. Maturitas 1986, 8(3):217-228.

2. Ho SC, Chan SG, Yip YB, Cheng A, Yi Q, Chan C: Menopausal symptoms and symptom clustering in Chinese women. Maturitas 1999, 33(3):219-227.

3. Fuh JL, Wang SJ, Lu SR, Juang KD, Chiu LM: The Kinmen women-health investigation (KIWI): a menopausal study of a population aged 40-54. Maturitas 2001, 39(2):117-124.

4. Sievert LL, Obermeyer CM, Saliba M: Symptom groupings at midlife: cross-cultural variation and association with job, home, and life change. Menopause 2007, 14(4):798-807.

5. Cray LA, Woods NF, Mitchell ES: Identifying symptom clusters during the menopausal transition: observations from the Seattle Midlife Women's Health Study. Climacteric 2013, 16(5):539-549. 
6. Greenblum CA, Rowe MA, Neff DF, Greenblum JS: Midlife women: symptoms associated with menopausal transition and early postmenopause and quality of life. Menopause 2013, 20(1):22-27.

7. Cray L, Woods NF, Mitchell ES: Symptom clusters during the late menopausal transition stage: observations from the Seattle Midlife Women's Health Study. Menopause 2010, 17(5):972-977.

8. Cray LA, Woods NF, Herting JR, Mitchell ES: Symptom clusters during the late reproductive stage through the early postmenopause: observations from the Seattle Midlife Women's Health Study. Menopause 2012, 19(8):864-869.

9. Terauchi M, Obayashi S, Akiyoshi M, Kato K, Matsushima E, Kubota T: Insomnia in Japanese peri- and postmenopausal women. Climacteric 2010, 13(5):479-486

10. Terauchi M, Obayashi S, Akiyoshi M, Kato K, Matsushima E, Kubota T: Effects of oral estrogen and hypnotics on Japanese peri- and postmenopausal women with sleep disturbance. J Obstet Gynaecol Res 2011, 37(7):741-749.

11. Terauchi M, Akiyoshi M, Owa Y, Kato K, Obayashi S, Kubota T: Effects of the Kampo medication keishibukuryogan on blood pressure in perimenopausal and postmenopausal women. Int J Gynecol Obstet 2011, 114(2):149-152.

12. Terauchi M, Hiramitsu S, Akiyoshi M, Owa Y, Kato K, Obayashi S, Matsushima E, Kubota T: Effects of three Kampo formulae: Tokishakuyakusan (TJ-23), Kamishoyosan (TJ-24), and Keishibukuryogan (TJ-25) on Japanese peri- and postmenopausal women with sleep disturbances. Arch Gynecol Obstet 2011, 284(4):913-921.

13. Terauchi M, Hiramitsu S, Akiyoshi M, Owa Y, Kato K, Obayashi S, Matsushima E, Kubota T: Associations between anxiety, depression and insomnia in peri- and post-menopausal women. Maturitas 2012, 72(1):61-65.

14. Terauchi M, Hiramitsu S, Akiyoshi M, Owa Y, Kato K, Obayashi S, Matsushima E, Kubota T: Associations among depression, anxiety and somatic symptoms in peri- and postmenopausal women. $J$ Obstet Gynaecol Res 2013, 39(5):1007-1013.

15. Terauchi M, Hiramitsu S, Akiyoshi M, Owa Y, Kato K, Obayashi S, Matsushima E, Kubota T: Effects of the kampo formula Tokishakuyakusan on headaches and concomitant depression in middle-aged women. Evid Based Complement Alternat Med 2014, 2014:593560.

16. Kravitz HM, Ganz PA, Bromberger J, Powell LH, Sutton-Tyrrell K, Meyer PM: Sleep difficulty in women at midlife: a community survey of sleep and the menopausal transition. Menopause 2003, 10(1):19-28.

17. Terauchi M, Horiguchi N, Kajiyama A, Akiyoshi M, Owa Y, Kato K, Kubota T: Effects of grape seed proanthocyanidin extract on menopausal symptoms, body composition, and cardiovascular parameters in middle-aged women a randomized, double-blind, placebo-controlled pilot study. Menopause 2014, 21(9):990-996.

18. Hunter M: The South-East England longitudinal study of the climacteric and postmenopause. Maturitas 1992, 14(2):117-126

19. Woods NF, Mariella A, Mitchell ES: Depressed mood symptoms during the menopausal transition: observations from the Seattle Midlife Women's Health Study. Climacteric 2006, 9(3):195-203.

20. Hall SA, Cinar A, Link CL, Kopp ZS, Roehrborn CG, Kaplan SA, Rosen RC Do urological symptoms cluster among women? Results from the Boston Area Community Health Survey. BJU Int 2008, 101(10):1257-1266.

21. Woods NF, Cray L, Mitchell ES, Herting JR: Endocrine biomarkers and symptom clusters during the menopausal transition and early postmenopause: observations from the Seattle Midlife Women's Health Study. Menopause 2014, 21(6):646-652.

22. Melby MK, Sievert LL, Anderson D, Obermeyer CM: Overview of methods used in cross-cultural comparisons of menopausal symptoms and their determinants: Guidelines for Strengthening the Reporting of Menopause and Aging (STROMA) studies. Maturitas 2011, 70(2):99-109.

23. Avis NE, Stellato R, Crawford S, Bromberger J, Ganz P, Cain V, Kagawa-Singer $\mathrm{M}$ : Is there a menopausal syndrome? Menopausal status and symptoms across racial/ethnic groups. Soc Sci Med 2001, 52(3):345-356.

doi:10.1186/s12905-014-0148-z

Cite this article as: Terauchi et al: Subgrouping of Japanese middle-aged women attending a menopause clinic using physical and psychological symptom profiles: a cross-sectional study. BMC Women's Health 2014 14:148.

\section{Submit your next manuscript to BioMed Central and take full advantage of:}

- Convenient online submission

- Thorough peer review

- No space constraints or color figure charges

- Immediate publication on acceptance

- Inclusion in PubMed, CAS, Scopus and Google Scholar

- Research which is freely available for redistribution

Submit your manuscript at www.biomedcentral.com/submit
Ciomed Central 